DOI 10. 18307/2017. 0104

(C) 2017 by Journal of Lake Sciences

\title{
鄱阳湖换水周期与示踪剂传输时间特征的数值模拟”
}

\author{
李云良 ${ }^{1}$, 姚 静 $^{1}$, 李梦凡 ${ }^{1,2}$, 张 奇 ${ }^{1,3}$ \\ (1: 中国科学院南京地理与湖泊研究所流域地理学重点实验室,南京 210008) \\ (2: 中国科学院大学, 北京 100049) \\ (3: 江西师范大学鄱阳湖湿地与流域研究教育部重点实验室,南昌 330022)
}

\begin{abstract}
摘 要: 在复杂湖泊水动力环境作用下,换水周期和传输时间变化直接影响着污染物的迁移和转化. 本文运用数值模拟 方法, 定量研究了季节水情动态下鄱阳湖换水周期和示踪剂传输时间的空间分布. 结果表明,不同季节下鄱阳湖换水周 期均具有较高的空间异质性, 贯穿整个湖区的主河道换水周期约 $<10 \mathrm{~d}$, 大多湖湾区的换水周期则长达 300 多天. 尽管不 同季节下换水周期空间分布格局几乎相似, 但受鄱阳湖水动力场的季节变化影响, 夏、秋季的换水周期要明显大于春、冬 季. 基于换水周期频率分布曲线的统计表明, $80 \%$ 的鄱阳湖区的换水周期约 $<30 \mathrm{~d}$,其余湖区换水周期为几十天至几百天, 表明鄱阳湖应该更加确切地描述为一个快速换水和慢速换水同时共存的湖泊系统. 鄱阳湖示踪剂传输时间介于 $4 \sim 32 \mathrm{~d}$, 夏、秋季的传输时间 (11 32d) 约为春、冬季 $(4 \sim 8 \mathrm{~d})$ 的 4 倍, 主要与鄱阳湖季节性水情特征及示踪剂的迁移路径有关. 本 文所获取的换水周期和示踪剂传输时间的时空分布信息可为今后鄱阳湖水质、水环境和生态系统管理和维护等方面提 供重要科学参考.
\end{abstract}

关键词: 换水周期;传输时间;空间异质性;染色示踪剂;水动力模型; 鄱阳湖

\section{Numerical simulations of residence time and tracer travel time in Lake Poyang}

\author{
LI Yunliang ${ }^{1}$, YAO Jing ${ }^{1}$, LI Mengfan ${ }^{1,2}$ \& ZHANG Qi ${ }^{1,3}$ \\ (1: Key Laboratory of Watershed Geographic Sciences, Nanjing Institute of Geography and Limnology, Chinese Academy of \\ Sciences, Nanjing 210008, P.R.China) \\ (2: University of Chinese Academy of Sciences, Beijing 100049, P.R.China) \\ (3: Key Laboratory of Poyang Lake Wetland and Watershed Research, Ministry of Education, Jiangxi Normal University, Nan- \\ chang 330022, P.R.China)
}

\begin{abstract}
Many physical, chemical and biological processes that occur within particular environments, including the transport of pollutants, sediments and other materials, are influenced by lake hydrodynamics. The residence time and travel time of these materials are most commonly used to represent the time scale of the physical transport processes and to elucidate associated water quality problems in lakes. The main objective of this study was to investigate the residence time and tracer travel time in Lake Poyang. A 2D hydrodynamic model (MIKE 21) was combined with dye tracer simulations (advection-dispersion equation) to determine the residence and travel time of the lake for various seasons. The results indicate that Lake Poyang exhibits strong but spatially heterogeneous residence time that varies with the different seasons. Generally, the average residence time is shorter than 10 days along the lake's main flow channels due to the prevailing northward flow pattern. Whereas the local topographically controlled flow patterns substantially increase, the residence time in most bays is approximately longer than 300 days with high spatial values. Flushing homogeneity curves show that approximately $80 \%$ of the lake area has the residence time varied between a few days and approximately 1 month, and around $20 \%$ of the area has the values in the range from several months to several hundred days. The results demonstrate that Lake Poyang is a mixing water system, which can be divided into fast and slow flushing systems in different seasons. Model simulations also reveal that the tracer travel time of Lake Poyang is in the range of 4-32 days across the lake. Depen-
\end{abstract}

* 国家重点基础研究发展计划项目 (2012CB417003)、江西省重大生态安全问题监控协同创新中心项目 (JXS-EW00 ) 和国家自然科学基金项目 (41401031,41371062,41301023) 联合资助. $2016-02-11$ 收稿; 2016-04-26 收修 改稿. 李云良(1983 ), 男, 助理研究员;E-mail: yunliangli@ niglas.ac.cn. 
ding on the seasonal water level regimes and associated pollutant transport pathways, the travel time from the released sources to the lake outlet Hukou during the summer and autumn (varies between 11-32 days) is four times greater than that under the spring and winter (i.e., 4-8 days). The outcomes of this study provide important information regarding the sustainable management of water quality, water environment and ecosystem for Lake Poyang.

Keywords: Residence time; travel time; spatial heterogeneous; dye tracer; hydrodynamic model; Lake Poyang

湖泊是全球陆地水系统中极为重要的组分, 因其具有丰富的地表水资源和高度的生态价值. 湖泊是人 们休闲娱乐、工农业用水和发展渔业的重要场所, 也是众多生物群赖以生存的必需环境 ${ }^{[1]}$. 湖泊大多位于流 域中下游地势低洼处, 起着过滤和缓冲的作用, 容纳了不同来源的水、沙和污染物等, 并将其转换为湖内的 物理、化学和生物过程等 ${ }^{[2]}$. 在湖泊水体复杂水动力环境作用下,换水周期和传输时间严重影响了湖区污染 物的迁移和转化,进而给湖泊水质的时空变化带来不容忽视的影响 ${ }^{\left[{ }^{[3]}\right.}$.

鄱阳湖是我国最大的淡水湖泊,也是长江中下游极具代表性的大型通江湖泊之一, 在优质淡水供给、洪 水调控和生物多样性保护等方面起着重要作用 ${ }^{[4]}$. 鄱阳湖水位的高度动态变化, 导致湖泊水体面积呈现年 内显著的萎缩与扩张,进而使得湖区洪旱灾害频发 ${ }^{[5]}$. 流域 “五河” 来水对湖泊水位起着主控作用,其次,鄱 阳湖与长江的水量交换也对湖泊水位造成季节性的顶托和拉空作用 ${ }^{[6-8]}$. 另外, 鄱阳湖也是全球重要生态 区, 承载着鄱阳湖生态经济区的可持续发展, 以占长江约 $16 \%$ 的年径流量影响着长江中下游供水和用水安 全 ${ }^{[9]}$. 但近些年来, 随着鄱阳湖周边工业化城镇化加快和人口的急剧增长, 污水和废水排放持续增加, 给鄱 阳湖水质造成严重威胁 ${ }^{[9]}$. 因此, 研究换水周期和污染物传输时间, 分析其影响因素, 可为鄱阳湖水质水环 境模拟、大型通江湖泊“江湖关系变化” 研究提供参考, 对保护 “一湖清水”、保障长江中下游水环境安全具 有重要现实意义.

文献调研发现, 先前研究 ${ }^{[4,10-11]}$ 基本都是采用一个平均意义上的比值来描述鄱阳湖换水周期, 即湖泊蓄 量 $(V)$ 和出流量 $(Q)$ 的比值 $(V / Q)$. 这种计算方法没有明确指明到底湖泊出流量和湖泊蓄量该如何取值以 及取值在哪个时间尺度 (星期、月还是年尺度) 上是比较可靠的? 先前研究几乎均得出较为一致的结论: 鄱 阳湖是一个快速的换水系统, 即换水时间很短. 由于不同学者所选取的典型年份水情特征有所不同, 导致鄱 阳湖换水周期的计算结果也差异较大, 大致变化范围由短则 10 天至长达 60 天 ${ }^{[4,10-11]}$. 这种基于比值的估算 方法比较适合于水文情势比较简单的湖泊系统, 但对于鄱阳湖而言, 因湖泊季节性水位变化幅度可达 $15 \mathrm{~m}^{[12]}$, 加之长江倒灌对鄱阳湖出流的频繁干扰 ${ }^{[7]}$, 无法确切量化与水位变化相关的湖泊出流量和湖泊蓄 量. 另外, 单一比值无法将换水周期与湖泊水环境因子 (例如温度、叶绿素 a 和电导率等) 的空间分布联系起 来, 导致其对湖泊水质水环境保护和借鉴方面所提供的科学参考价值尚显不足 ${ }^{[13]}$. 实际上, 基于单一比值 的换水周期计算只是对湖泊换水能力的一般性描述, 无法反映不同时期与不同湖区的换水能力状况 ${ }^{[13]}$, 而 这些又是当今大尺度湖泊系统研究所关注的重要方面. 客观而言, 换水周期具有时间上显著变化和空间上 的固有异质性, 因而寻求一种更加合理可靠的方法开展鄱阳湖换水周期的空间调查及其对季节变化的响应 是当前研究的一个重要任务.

基于上述原因,结合鄱阳湖目前水质水环境问题所面临的严峻考验, 本文在先前构建的鄱阳湖二维水 动力模型的基础上耦合染色示踪剂方法, 开展鄱阳湖不同季节的换水周期和污染物 (示踪剂) 传输时间的模 拟研究, 期望能对水力联系复杂的鄱阳湖水系统有更加全面和清晰的认识. 本文主要解决以下几个问题或 研究目标: (1) 鄱阳湖换水周期是否有明显的空间差异? 其空间分布格局是否随着不同季节水情变化而差 异显著? (2) 示踪剂传输时间在季节变化的空间尺度上有多大变化和差异? (3) 换水周期和示踪剂传输时 间与水流空间结构有怎样的联系? 这些问题的进一步明确不仅有助于对湖泊空间水动力场的深人认识, 也 可为湖区污染物的输移模拟和空间分布提供科学参考, 更重要的是可用于进一步阐释鄱阳湖水环境的空间 变化及其响应机制.

\section{1 研究方法}

\section{1 研究区介绍}

鄱阳湖 ( $28^{\circ} 24^{\prime} \sim 29^{\circ} 46^{\prime} \mathrm{N}, 115^{\circ} 49 \sim 116^{\circ} 49^{\prime} \mathrm{E}$ ) 位于江西省北部及长江中下游南岸, 南北长约 $173 \mathrm{~km}$, 东 
西最大宽度约 $74 \mathrm{~km}$, 北部人江水道最窄处的宽度约 $2.8 \mathrm{~km}$, 湖岸线总长约 $1200 \mathrm{~km}$ (图 1). 湖盆高程总体呈南 高北低的趋势, 最大高程差可达 $13 \mathrm{~m}$, 南、北部平均高程相差约 $6.5 \mathrm{~m}$ (图 1 ). 鄱阳湖大致包括主河道、岛屿、 洪泛洲滩等典型地貌. 根据先前计算结果, 洪水季节约 $85 \%$ 的鄱阳湖区平均水深小于 $6 \mathrm{~m}$, 最大水深可达 $30 \mathrm{~m}^{[12]}$. 湖区气象条件空间差异较小, 主要表现在季节性变化, 多年平均降雨量和蒸发量分别为 1766 和 $1236 \mathrm{~mm}^{[4]}$. 根据实测资料统计的湖区年风向以 $\mathrm{NE}$ 向为主, 年内平均风速约为 $3.03 \mathrm{~m} / \mathrm{s}^{[14]}$. 鄱阳湖主要承 纳赣江、抚河、信江、饶河与修水 5 大水系来水, 经调蓄后注人长江, 其与 “五河” 及长江之间频繁的水量交换 形成了鄱阳湖高动态的水位和水面积变化. 根据流量资料统计得出, 竷江流域人湖径流约占年人湖总量 $62 \%$ 、抚河流域约占 $11 \%$ 、信江流域约占 $16 \%$ 、饶河流域和修水流域分别约占 $8 \%$ 和 $3 \%{ }^{[15]}$. 鄱阳湖洪、枯季 节的水位差可达 $15 \mathrm{~m}$, 枯水季节的空间水位差可达 $5 \mathrm{~m}$, 而洪水季节整个湖区的水位基本保持一致 ${ }^{[12]}$. 丰水 期鄱阳湖水面面积可达 $3000 \mathrm{~km}^{2}$, 而枯水期萎缩至不足 $1000 \mathrm{~km}^{2[16]}$, 呈现 “洪水一片, 枯水一线” 的独特洪 泛特征.

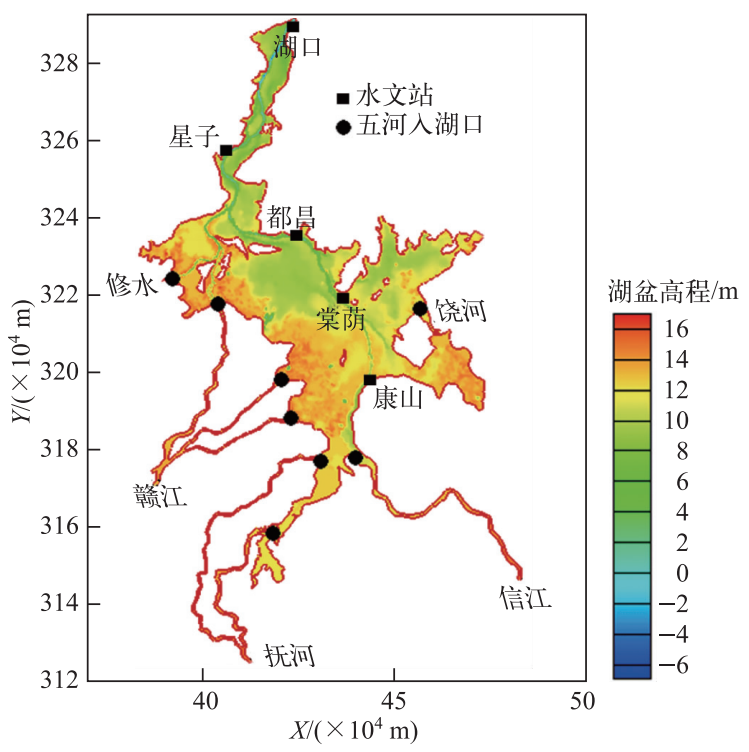

图 1 鄱阳湖湖盆地形、水文站点及水系分布

Fig.1 Lake bathymetry, hydrological gauging stations and major inflow rivers of Lake Poyang

\section{2 水动力模型与构建}

鄱阳湖水动力模型采用丹麦水力研究所 (DHI) 开发的 MIKE 21, 该模型属于平面二维水流数学模型. MIKE 21 模型已经成功应用于不同国家及地区的河流、湖泊和海湾等大型地表水体的流场模拟, 详细模型理 论与方法, 本文不再赘述. 鄱阳湖水动力模型计算域范围及岸线边界根据湖泊历史洪水淹没范围确定 (包括 关键河段的河道模拟; 图 1), 约占全鄱阳湖流域面积的 $2 \%$. 模型采用三角形网格来适应鄱阳湖复杂的湖盆 地形和岸线, 对主河道区域进行网格整体细化剖分, 而远离主河道的洪泛洲滩及岸线周边区域采用粗化的 网格剖分. 为了保证湖区的水量平衡, 模型将 5 个站点 (图 1 ) 的日降雨和蒸发资料考虑到水动力模型中参 与计算. 尽管风并不是鄱阳湖水动力场的主要驱动因子, 模型将定常风纳人到水动力模型考虑其对局部湖 区流速的影响 ${ }^{[14]}$. 流域 “五河” 的人湖径流过程作为水动力模型上游开边界条件 (模型会根据河段上游的输 人流量进行河道水流模拟), 江湖交汇处的湖口水位过程作为下游开边界条件, 以此表征流域五河-鄱阳湖长江之间的相互作用. 模型所需率定的主要参数包括曼宁粘率系数和涡粘系数. 空间变化的曼宁数 $M$ 给定 为 $30 \sim 50 \mathrm{~m}^{1 / 3} / \mathrm{s}$, 分别适应于湖泊底部的洪泛洲滩和主河道, 浴粘系数采用 Smagorinsky 公式计算, 其中子浴 扩散系数 $C_{\mathrm{s}}$ 取 0.28 . 鉴于鄱阳湖水动力模型已成功构建并广泛应用于鄱阳湖水动力研究 ${ }^{[8,12,14,17-21]}$, 本文仅 将模型的关键信息和模型构建的重要方面汇总于表 1 . 
表 1 鄱阳湖水动力模型 MIKE 21 的构建和参数设定

Tab. 1 Construction and parameters in the MIKE 21 hydrodynamic model of Lake Poyang

\begin{tabular}{lcc}
\hline 模型构建 & 模型给定 & 参考文献 \\
\hline 模型适用性 & 正压模式,深度平均 $(2 \mathrm{D})$ & {$[12,20]$} \\
上游开边界 & 流域五河人湖日径流过程线 & {$[12]$} \\
下游开边界 & 湖口日水位过程线 & {$[12,18]$} \\
湖区降雨/蒸发 & 空间均一,但随时间变化 & {$[12]$} \\
湖区风场 & 空间定常风 $\mathrm{NE}=45^{\circ}$, 风速 $=3.03 \mathrm{~m} / \mathrm{s}$ & {$[14,19-20]$} \\
湖盆地形 & 更新到 2000 年 & {$[8]$} \\
空间计算域 & 约 $3200 \mathrm{~km}^{2}$ & {$[12]$} \\
离散分辨率 & 网格尺寸变化范围为 $70 \sim 1500 \mathrm{~m}$ & {$[12]$} \\
时间步长 & 变时间步长为 $5 \sim 3600 \mathrm{~s}$ & {$[12]$} \\
CFL 条件数 & 设定为 1.0 & {$[12]$} \\
曼宁徏率系数 & 空间分布,变化范围为 $30 ~ 50 \mathrm{~m}^{1 / 3} / \mathrm{s}$ & {$[8,12,17,21]$} \\
涡粘系数 & 空间取均一值 0.28 & {$[12,14]$} \\
\hline
\end{tabular}

\section{3 模型率定与验证}

鄱阳湖水动力模型 MIKE 21 已进行了全面的率定和验证, 主要是集中于 2000-2010 年的连续年份验 证和典型年份验证 ${ }^{[12,14,20]}$, 通过 Nash-Sutcliffe 效率系数、确定性系数和相对误差来评价湖泊水位、湖泊水面 积、湖泊出流量和流速等关键变量的模拟效果 ${ }^{[12]}$. 水位验证采用星子、都昌、棠荫和康山(图 1) 4 个站点 $2000-$ 2008 年的日水位, 各站点拟合的 Nash-Sutcliffe 效率系数可达 0.98 , 相对误差均在 $\pm 5 \%$ 以内 ${ }^{[12]}$; 选取 2004 年 的 14 景 MODIS 影像来验证水动力模型,洪水期和枯水期水面积拟合的相对误差分别为 $3.3 \%$ 和 $16.8 \%^{[12]}$; 鄱阳湖水动力模型能够很好地模拟 2003-2008 年湖泊出流和长江倒灌量时序变化, 拟合的确定性系数可达 0.92 , 相对误差约为 $-12.9 \%{ }^{[12]}$; 通过 2008 年实测的湖口和星子 ADCP 断面流速走航资料来验证水动力模 型, 结果表明 MIKE 21 模型能够理想地再现鄱阳湖洪枯季节的水流变化, 流速拟合的确定性系数可达 0.80 以上 ${ }^{[20]}$. 此外, MIKE 21 模型能够成功捕捉 2005 年季节水情变化下鄱阳湖典型湖区的水动力场特征 ${ }^{[12]}$. 本 文限于篇幅, 不再过多陈述 MIKE 21 水动力模型的率定和验证情况, 具体可参考该模型在鄱阳湖水文水动 力研究方面先前发表的成果 ${ }^{[12,14,19-20]}$.

\section{4 换水周期与传输时间定义}

不同学者对湖泊换水能力从不同角度进行了定义, 如水龄、水力滞留时间和换水周期等 ${ }^{[22]}$. 本文采用 换水周期 ( $e$-folding time) 这一应用比较广泛的概念来定量研究鄱阳湖的换水能力,即换水周期计算采用基 于浓度变化的指数衰减函数来表示 ${ }^{[23-25]}$ :

$$
C_{t}=C_{0} \cdot \mathrm{e}^{-t / T_{t}}
$$

式中, $t$ 表示时间, $C_{0}$ 表示示踪剂初始浓度值, $C_{t}$ 表示 $t$ 时刻的剩余示踪剂浓度值. 由公式(1) 可得, 当 $t=T_{\mathrm{f}}$ $(V / Q)$ 时, 浓度已经衰减到初始浓度的 $\mathrm{e}^{-1}$ 或 37\% . 因此, 换水周期定义为剩余浓度降低至初始浓度的 37\% 时所需要的时间.

示踪剂传输时间 (travel time) 是另一个表征湖泊换水能力的重要指标, 其通常定义为一种给定浓度的保 守型示踪剂从它的起始投放位置到某一观测位置出现浓度峰值时所需要的时间 ${ }^{[26-28]}$. 当某一观测位置出现 两个或多个浓度峰值时, 通常将最大浓度所对应的时间定义为湖泊系统的示踪剂传输时间 ${ }^{[29]}$.

通过上述定义可知, 换水周期和传输时间既有联系又有一定差异, 两者均是基于污染物迁移而提出的 与湖泊水流运动密切相关的重要指标,但两者却是从不同角度来揭示湖泊系统的换水能力.

\section{5 模拟方案与模型设置}

本文通过水动力模型 MIKE 21 耦合对流-弥散方程 ${ }^{[30-31]}$ 来模拟计算鄱阳湖换水周期和示踪剂传输时 间. 根据上述定义, 为了详细调查鄱阳湖换水周期的空间异质性, 本文的研究策略是将整个鄱阳湖水体染成 单位浓度为 1 的保守型示踪剂, 通过监测水动力模型每个网格单元的剩余浓度变化,便可获得鄱阳湖换水 
周期的空间分布. 因鄱阳湖污染物的主要来源为流域 “五河” 水系, 本文选取 3 个人湖口作为主要投放区域 (代表西部、南部和东部湖区) 来探究空间尺度上的鄱阳湖示踪剂传输时间, 并在湖口断面观测浓度的响应 与变化.

因为鄱阳湖水情年际变化较大, 一个平均年份的模拟计算要比典型年份更具有普适性. 因此, 本文将 2001-2010 年(共 10 年) 的平均条件作为先前 MIKE 21 模型的基础输人,即模拟时间设定为一个完整的水 文年 (365 d). 模型更新输人主要包括上游“五河”开边界条件、湖口水位边界条件和湖区上边界气象条件, 而其他输人和模型参数保持不变 (表 1). 对于换水周期计算, 将初始空间浓度场设定为单位浓度 1 (本文浓 度单位设定为 $\mathrm{kg} / \mathrm{m}^{3}$ ), “五河” 开边界浓度值设定为 0 , 湖口开边界处设定为浓度自由出流边界; 对于示踪剂 传输时间计算, 将初始空间浓度场设定为 0,3 个代表性投放点分别设定为单位浓度 1 , “五河” 开边界浓度值 设定为 0 , 湖口开边界处设定为浓度自由出流边界. 为了进一步研究不同季节下的换水周期和示踪剂传输时 间变化,本文模拟将染色示踪剂 (初始浓度场和点源) 的投放时间分别设定为 4 月 1 日 (涨水期)、7 月 1 日 (洪水期)、 10 月 1 日 (退水期) 和 1 月 1 日 (枯水期), 相应的模拟结束时间分别为次年的 4、7、10 和 1 月 (通 过平均水文年资料来插补次年对应月份的序列资料), 以此综合表征鄱阳湖春、夏、秋和冬季的换水能力.

\section{2 结果与分析}

\section{1 换水周期模拟}

换水周期模拟时鄱阳湖北部人江通道湖口、星子站的浓度曲线随时间变化大体呈指数衰减形式,充分 表明本文所采用的换水周期计算方法 (式 (1)) 在鄱阳湖具有很好的适用性,保证了模型模拟结果的合理性 与可靠性. 模拟结果表明, 春、冬季节湖口和星子站的换水周期约为 $10 \mathrm{~d}$ 左右, 而夏、秋季两个典型点位附近 水体的换水周期变化范围为 $20 \sim 30 \mathrm{~d}$, 尤其是夏季的换水周期可达 $30 \mathrm{~d}$ (图 2). 为了深人分析鄱阳湖不同季 节的换水周期空间分布,这里对 4 个季节的模拟结果分别加以分析与探讨. 由图 3a 可以发现,整个湖区的 换水周期较快, 大部分湖区换水时间约小于 $10 \mathrm{~d}$, 邻近西部湖岸线的少部分湖区、东北部和东南部两大湖湾 区有着相对较长的换水周期,平均换水时间约为 $30 \mathrm{~d}$. 总体来看, 春季鄱阳湖的换水周期空间异质性较弱, 主要是因为该季节强大的流域 “五河” 来水导致了整个鄱阳湖快速变化的湖流,换水时间也相对较短. 夏天 鄱阳湖换水周期的空间异质性较强, 换水周期从北到南大致呈梯度变化, 北部湖区换水周期变化范围约 $30 \sim 60 \mathrm{~d}$, 湖区中游小部分湖区换水周期约为 $10 \sim 20 \mathrm{~d}$, 而南部湖区的换水周期则少于 $10 \mathrm{~d}$ (图 3b). 换水周 期的梯度分布主要与鄱阳湖洪水期水情变化密切相关, 该时期鄱阳湖出流受到长江顶托而整体水面保持水 平, 此时鄱阳湖北部湖区受长江影响较为显著, 而上游流域的 “五河” 入湖径流则加快了南部湖区水体的换 水能力. 由图 $3 \mathrm{~b}$ 还可以看出, 东北部和东南部湖湾区和最南部湖湾区的换水周期较长, 换水时间长达 $180 \mathrm{~d}$, 最高值可达 300 多天, 表明这些局部湖区受自身湖盆形态和水动力影响其换水能力很弱, 需要长时间才能 完成一次换水. 从图 3c 不难发现, 秋季换水周期的空间分布格局与夏季呈现很好的一致性. 同夏季相比,秋 季鄱阳湖换水周期整体上较短, 北部至中部广大湖区的换水周期约 $10 \sim 20 \mathrm{~d}$, 而南部和西部湖区换水周期约小 于 $10 \mathrm{~d}$. 同样可以发现, 东北部、东南部和最南部湖湾区的换水周期仍然相对较长, 换水时间最长可达 $240 \mathrm{~d}$. 冬季是鄱阳湖的低枯水位时期, 鄱阳湖水体面积严重萎缩, 除了贯穿整个湖区的主河道, 此时的鄱阳湖已被 分割成许多相对独立的小型水体, 换水周期也相应呈现较高的空间异质性 (图 3d). 此时, 主河道的换水周 期相对较短, 约为 $7 \mathrm{~d}$, 相对独立的碟形湖或者小型水体因湖水位较低无法与主湖区进行充分的水量交换, 换水周期相对较长, 约 $20 \sim 30 \mathrm{~d}$, 而临近湖岸线的大部分湖湾区换水周期长达 $120 \mathrm{~d}$ 左右.

总体而言, 鄱阳湖换水周期在不同季节均具有较为明显的空间异质性. 尽管换水周期的空间值随季节 变化也呈现出较大的差异性, 但换水周期的空间分布格局在季节尺度上具有高度相似性和一致性. 空间上, 主河道换水周期整体上约小于 $10 \mathrm{~d}$, 洪泛洲滩等广大浅水区域的换水周期随季节水情约变化为几十天到几 个月, 大多数湖湾区的换水周期变化为几个月至几百天, 最长换水周期可达 300 多天. 在不同季节的水情影 响下, 鄱阳湖主河道有着大致沿向北方向运动的快速水流 (最大流速可达 $1.0 \mathrm{~m} / \mathrm{s}$ ), 而远离主河道的洪泛洲 滩因水流形态复杂其流速相对平缓, 而近湖岸的一些湖湾区流速极其缓慢 (约 $<0.1 \mathrm{~m} / \mathrm{s}$ ), 加上风在这些局 部湖区的显著作用, 经常伴有环流发生 ${ }^{[12,14]}$. 鄱阳湖的季节性洪泛变化是其不同于其他湖泊的重要特征之 

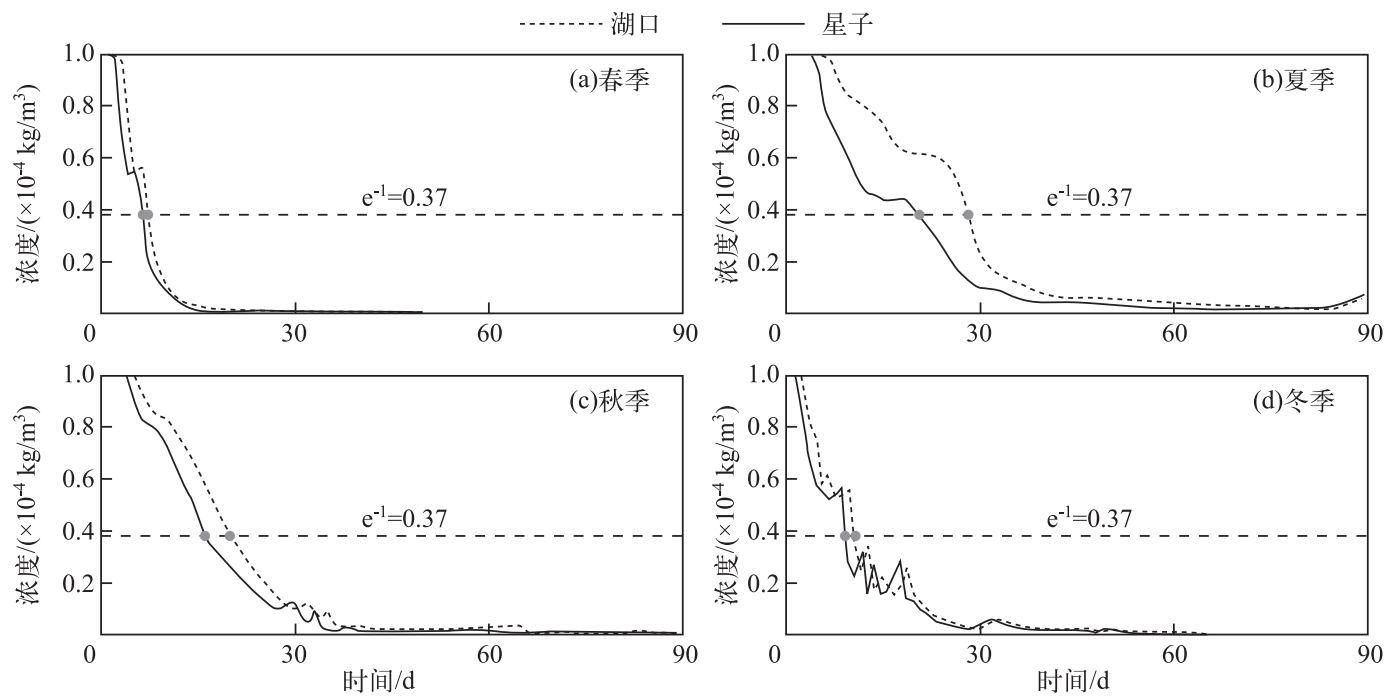

图 2 湖口和星子站染色示踪剂浓度随时间变化曲线(圆点表示 $37 \%$ 浓度值对应的换水周期)

Fig.2 Temporal variations in dye tracer concentrations at Hukou and Xingzi gauging stations of Lake Poyang

( The circle represents the residence time corresponding to $37 \%$ of the initial concentration)

一, 因夏、秋季节鄱阳湖呈现典型“湖相”特征, 除了流域“五河” 入湖口外, 湖区流速整体较小 (图 4b 和 c ), 而春、冬季明显的 “河相” 特征导致了湖泊主河道内快速变化的束窄水流, 整个鄱阳湖的流速相对较大 (图 $4 \mathrm{a}$ 和 $\mathrm{d}$ ), 这也是湖区夏、秋季的换水周期要明显高于春、冬季的主要原因. 由此表明,水动力场特征是决定 换水周期空间分布格局的重要因素. 换言之, 在流域和长江来水的外部复合作用下, 鄱阳湖换水周期的空间 分布格局主要与自身湖盆地形(图 1) 和内部水动力场(图 4)密切相关.

\section{2 频率分布曲线}

基于频率分布曲线 (Flushing homogeneity curves)的变异性分析主要是用来反映换水周期在整个湖泊系 统内的面积分布状况 ${ }^{[32]}$, 以此来深人理解湖泊系统的换水能力. 一般而言,该曲线形态变化越陡峭, 表示湖 泊系统换水周期变异性越弱; 反之, 表示湖泊系统换水周期的变异性越强. 根据频率分布曲线定义及其变异 性表征,一个快速的换水系统应当具有沿垂向变化陡峭的频率分布曲线 ${ }^{[32]}$. 统计结果显示, 在不同季节下, 鄱阳湖换水周期频率分布曲线都存在一个明显拐点,约 $80 \%$ 的鄱阳湖区换水周期 $<30 \mathrm{~d}$, 而换水周期 $>30 \mathrm{~d}$ 的湖区面积所占比例不足或者远低于 $20 \%$ ( 图 5). 该频率分布曲线大体上由陡峭变化过渡至颇为平缓的变 化态势 (图 5 箭头所示) , 由此表明鄱阳湖换水周期具有较高的空间异质性,这也与先前的模拟结果一致(图 3). 尽管鄱阳湖大部分湖区的换水时间较短,但基于频率分布曲线的变异性分析表明鄱阳湖并不能简单定 义或描述为一个换水快的湖泊系统 (图 5). 从全湖区来说, 鄱阳湖应该更加确切地描述为一个快速换水和 慢速换水在空间上共存的湖泊系统. 根据换水周期的空间模拟结果, 应将主河道和湖湾区等换水周期差异 明显的湖域加以区分和分别对待.

\section{3 示踪剂传输时间模拟}

3 个典型释放点 $\mathrm{C} 1 \sim \mathrm{C} 3$ 的示踪剂传输时间在季节变化上有较大差异 (图 6). 模拟结果表明, 鄱阳湖春 季的示踪剂传输时间约 $<7 \mathrm{~d}$, 夏季和秋季的示踪剂传输时间约为 $11 \sim 32 \mathrm{~d}$, 冬季的示踪剂传输时间与春季相 差不大, 约为 4 $8 \mathrm{~d}$. 就同一个释放点而言, 夏、秋季释放示踪剂到达湖口所需传输时间约为春、冬季的 4 倍 (图 6). 根据水动力场变化特征(图 4) 分析得出, 夏、秋季整个鄱阳湖的水位相对较高, 湖区水流运动较慢, 导致染色示踪剂的对流和弥散进程也相对迟缓, 示踪剂到达湖口所需的时间较长; 春、冬季翻阳湖空间上水 面坡降相对较大, 快速运动的水流加速了示踪剂达到湖口所需的时间. 就空间上 3 个释放点而言, C1 释放点 因距离湖口相对较近, 沿主河道的水流运动路径也相对较短, 致使其水力传输时间也相对较短, 而距离湖口 

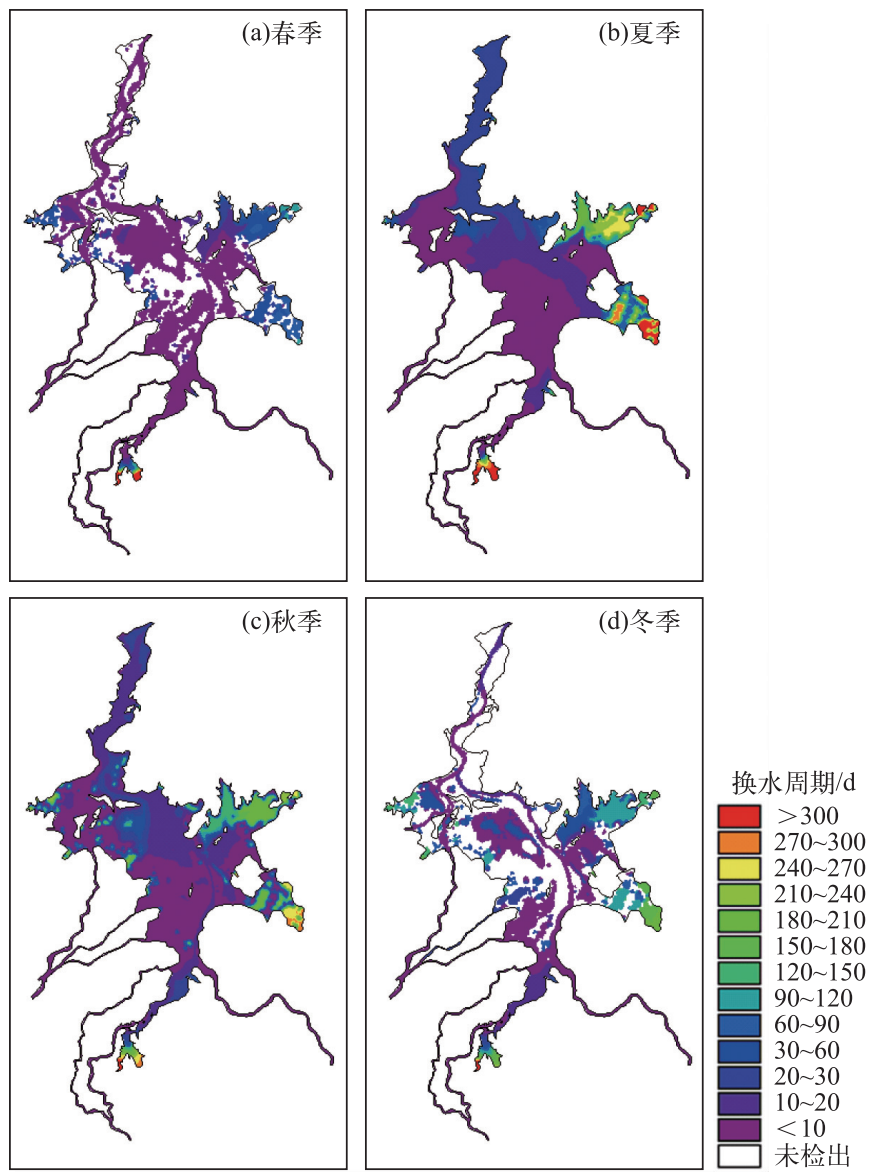

图 3 鄱阳湖季节性换水周期的空间分布

Fig.3 Spatial distribution of residence time in different seasons of Lake Poyang

相对较远的 C2 释放点的传输时间相对较长 (图 6). 由此表明, 示踪剂到达湖口所需的传输时间还与示踪剂 的迁移路径和距离有关,这也与先前的示踪剂运动轨迹模拟结果基本一致 ${ }^{[20]}$.

\section{3 讨论与展望}

本文采用一个完全物理机制的二维水动力模型来首次开展鄱阳湖换水周期和示踪剂传输时间的空间 模拟,但基于浓度变化的模拟结果还无法通过原位监测来进行有效验证. 本文所开展研究是先前鄱阳湖水 动力和污染物输移耦合模型 ${ }^{[19-20]}$ 的细化模拟和进一步应用, 研究重点从湖区水流的空间格局拓展到换水能 力的空间异质性调查. 已有不少研究充分表明空间尺度上的换水周期和污染物传输时间通常与水环境参数 之间存在极显著的相关性, 进而对湖泊生态环境有着重要的指示意义 ${ }^{[33]}$. 已有研究 ${ }^{[34]}$ 认为鄱阳湖水体叶绿 素 $\mathrm{a}$ 高浓度分布区主要集中在东部湖湾区, 而相对低浓度分布区大致位于主河道等快速水流区,这种空间 分布格局与本文换水周期的空间分布具有高度一致性,这在一定程度上印证了本文模拟结果的可靠性. 此 外,一些其他水环境参数 (总氮、总磷和高锰酸盐指数) 的空间调查及其分布格局 ${ }^{[35]}$ 总体上与之相似, 进一 步证实了鄱阳湖换水周期和水环境参数之间的密切联系. 在鄱阳湖水质逐渐恶化以及湖区富营养化趋势逐 步发展的背景下,本文所获取的换水周期和水力传输时间的空间分布信息可为今后鄱阳湖水质、水环境和 生态系统管理和维护等方面提供重要科学参考价值.

本文借助情景假定方法, 基于水动力模型耦合示踪剂对流-弥散方程, 较系统地评估了平均意义上的鄱 

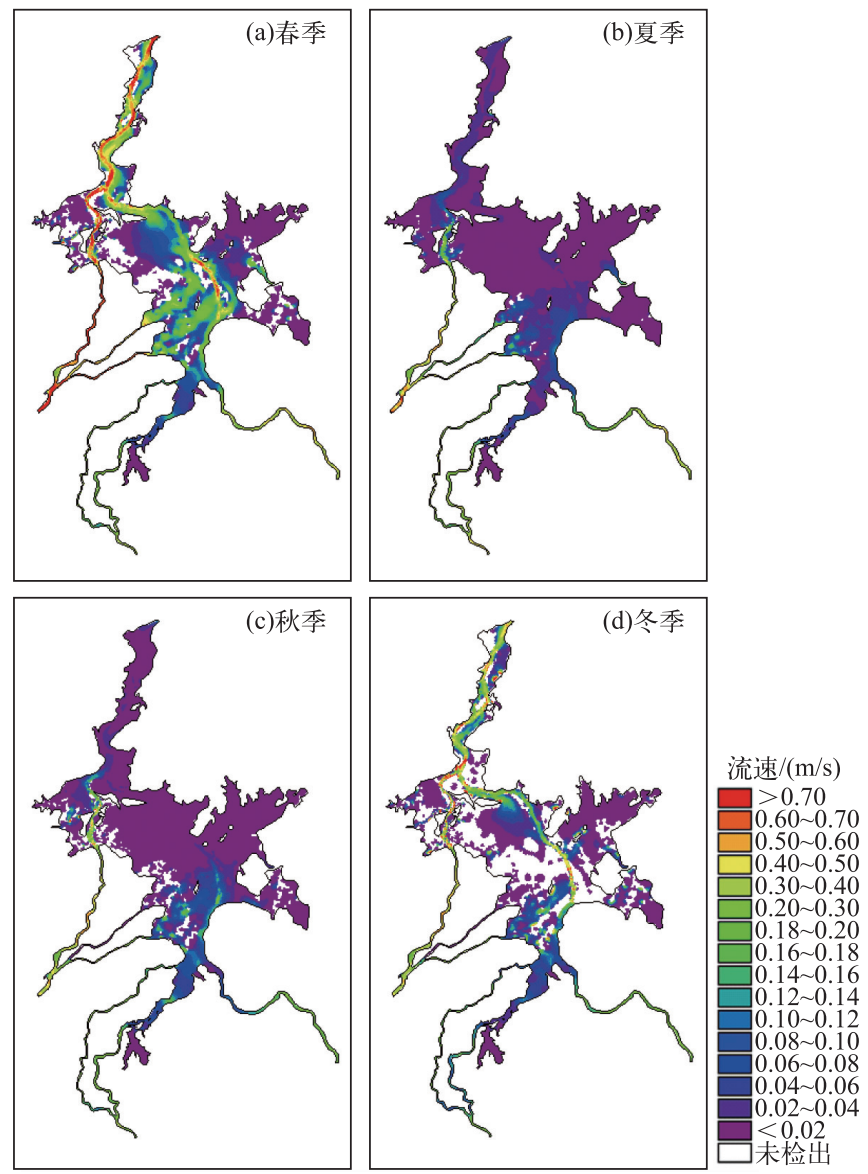

图 4 基于深度平均的鄱阳湖季节性流速场特征

Fig.4 Depth-averaged velocity fields in different seasons of Lake Poyang

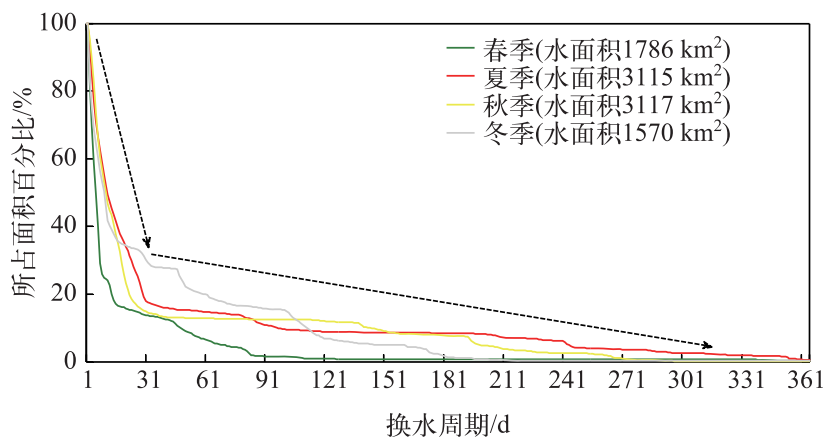

图 5 鄱阳湖换水周期变异性分析的频率分布曲线

Fig.5 Flushing homogeneity curves of residence time in different seasons of Lake Poyang

阳湖换水周期和示踪剂传输时间的时空变化特征,使我们对鄱阳湖换水能力有了全新和深人的认识,但流 域“五河” 和长江水情变化对湖泊换水能力的影响和贡献仍没有进一步区分,该工作对未来拟建鄱阳湖水力 枢纽工程的潜在影响具有重大实际意义, 这将是下一步研究工作的重点. 另外, 未来研究还应该聚焦于鄱阳 

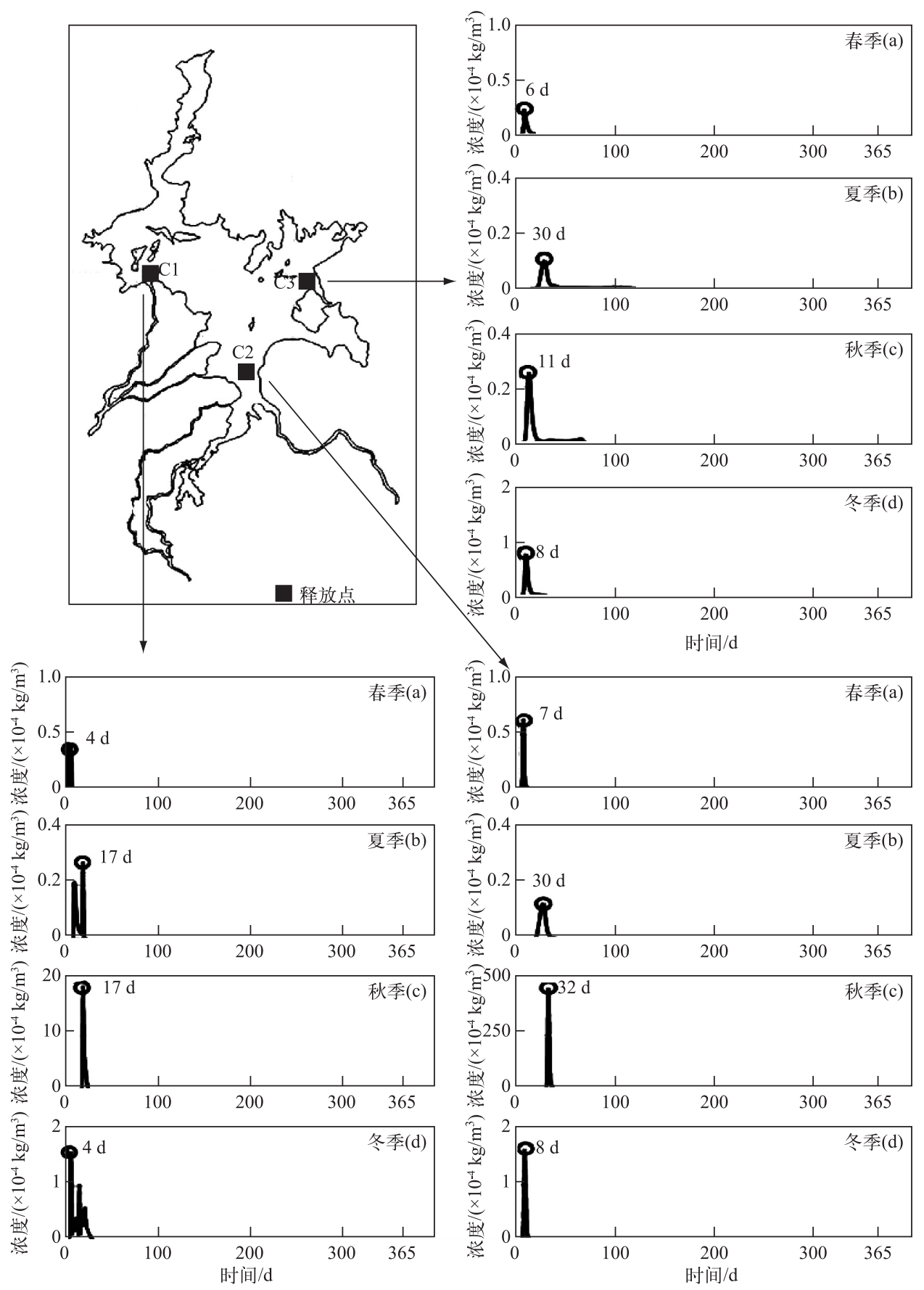

图 6 不同季节湖口站染色示踪剂浓度时间变化曲线及水力传输时间 (圆圈表示峰值浓度对应的传输时间)

Fig.6 Time series of dye tracer concentrations at Hukou gauging station outlet and associated hydraulic travel time in four seasons of Lake Poyang (The circle represents the travel time corresponding to the peak concentration)

湖出现水质恶化征兆的典型湖湾区, 例如东北部湖湾区 ${ }^{[36]}$. 


\section{4 结论}

本文基于二维水动力模型 MIKE 21 耦合对流-弥散方程, 首次针对一个洪泛湖泊系统开展换水周期和 示踪剂传输时间研究, 引人染色示踪剂的概念来设置不同的模拟情景, 主要贡献是揭示了季节变化下鄱阳 湖换水周期和示踪剂传输时间的空间分布及其影响因素,为水环境状况日益恶化的鄱阳湖及其今后健康发 展提供重要科学依据和参考. 主要得出以下几点结论:

1) 总体上,鄱阳湖换水周期具有较高的空间异质性. 在季节变化下,水流运动较快的主河道的换水周期 约 $<10 \mathrm{~d}$,一些水流运动相对缓慢的湖湾区的换水周期长达 300 多天. 夏、秋季节的换水周期要明显大于春、 冬季节,主要受季节水情作用下的鄱阳湖水动力场变化影响.

2) 基于频率分布曲线的换水系统变异性分析结果表明,鄱阳湖不能简单定义为一个快速换水系统. 鉴 于 $80 \%$ 的鄱阳湖区换水周期约 $<30 \mathrm{~d}$, 以及 $20 \%$ 的湖区换水周期约为几十天至几百天, 鄱阳湖应该更加确切 地描述为一个快速换水和慢速换水同时共存的湖泊系统,以充分反映湖区换水周期的空间差异.

3 ) 空间上, 鄱阳湖示踪剂传输时间变化范围介于 4 32 d, 但夏、秋季的传输时间 (11 32 d ) 大约为春、 冬季 $(4 \sim 8 \mathrm{~d})$ 的 4 倍, 主要与鄱阳湖季节性水情特征及示踪剂的迁移路径有关.

\section{5 参考文献}

[ 1 ] Lehner B, Döll P. Development and validation of a global database of lakes, reservoirs and wetlands. Journal of Hydrolo$g y, 2004,296(1 / 2 / 3 / 4): 1-22$.

[ 2 ] Ji Zhengang ed. Hydrodynamics and water quality. Modeling rivers, lakes and estuaries. Beijing: China Ocean Press, 2012. [季振刚. 水动力学和水质-河流、湖泊及河口数值模拟. 北京: 海洋出版社, 2012.]

[ 3 ] Pilotti M, Simoncelli S, Valerio G. A simple approach to the evaluation of the actual water renewal time of natural stratified lakes. Water Resources Research, 2014, 50(4) : 2830-2849.

[ 4 ] Wang Sumin, Dou Hongshen eds. Chinese lakes. Beijing: Science Press, 1998. [王苏民, 窦鸿身. 中国湖泊志. 北京: 科学出版社, 1998.]

[ 5 ] Shankman D, Heim BD, Song J. Flood frequency in China's Poyang Lake region: Trends and teleconnections. International Journal of Climatology, 2006, 26: 1255-1266.

[ 6 ] Hu Q, Feng S, Guo H et al. Interactions of the Yangtze River flow and hydrologic processes of the Poyang Lake, China. Journal of Hydrology, 2007, 347(1/2): 90-100.

[ 7 ] Guo H, Hu Q, Zhang Q et al. Effects of the Three Gorges Dam on Yangtze River flow and river interaction with Poyang Lake, China: 2003 - 2008. Journal of Hydrology, 2012, 416/417: 19-27.

[ 8 ] Zhang Q, Ye XC, Werner AD et al. An investigation of enhanced recessions in Poyang Lake: Comparison of Yangtze River and local catchment impacts. Journal of Hydrology, 2014, 517: 425-434.

[ 9 ] Li X, Zhang L, Yang G et al. Impacts of human activities and climate change on the water environment of Lake Poyang Basin, China. Geoenvironmental Disasters, 2015, 2: 1-12.

[10] Wu Z, Lai X, Zhang L et al. Phytoplankton chlorophyll a in Lake Poyang and its tributaries during dry, mid-dry and wet seasons: A 4-year study. Knowledge and Management of Aquatic Ecosystems, 2014, 412: 1-13.

[11] Zhu Haihong, Zhang Ben eds. Poyang Lake. Hefei : Press of University of Science and Technology of China, 1997. [ 朱海 虹, 张本. 鄱阳湖. 合肥: 中国科学技术大学出版社, 1997.]

[12] Li YL, Zhang Q, Yao J et al. Hydrodynamic and hydrological modeling of Poyang Lake-catchment system in China. Journal of Hydrologic Engineering, 2014, 19(3): 607-616.

[13] Shaha DC, Cho YK, Seo GH et al. Using flushing rate to investigate spring-neap and spatial variations of gravitational circulation and tidal exchanges in an estuary. Hydrology and Earth System Sciences, 2010, 14: 1465-1476.

[14] Yao Jing, Zhang Qi, Li Yunliang et al. The influence of uniform winds on hydrodynamics of Lake Poyang. J Lake Sci, 2016, 28(1) : 225-236. DOI: 10.18307/2016.0126. [姚静, 张奇, 李云良等. 定常风对鄱阳湖水动力的影响. 湖泊 科学, $2016,28(1): 225-236$. ]

[15] Li Yunliang, Zhang Qi, Li Miao et al. Using BP neural networks for water level simulation in Poyang Lake. Resources and 
Environment in the Yangtze Basin, 2015, 24(2) : 233-240. [李云良, 张奇, 李沝等. 基于 BP 神经网络的鄱阳湖水位 模拟. 长江流域资源与环境, 2015, 24(2): 233-240.]

[16] Feng L, Hu C, Chen X et al. Assessment of inundation changes of Poyang Lake using MODIS observation between 2000 and 2010. Remote Sensing of Environment, 2012, 121: 80-92.

[17] Li Yunliang, Zhang Qi, Yao Jing et al. Integrated simulation of hydrological and hydrodynamic processes for Lake Poyangcatchment system. J Lake Sci, 2013, 25(2) : 227-235. DOI: 10.18307/2013.0208. [李云良, 张奇, 姚静等. 鄱阳湖湖 泊流域系统水文水动力联合模拟. 湖泊科学, 2013, 25(2): 227-235.]

[18] Li YL, Zhang Q, Werner AD et al. Investigating a complex lake-catchment-river system using artificial neural networks: Poyang Lake (China). Hydrology Research, 2015, 46(6) : 912-928.

[19] Li YL, Zhang Q, Yao J. Investigation of residence and travel time in a large floodplain lake with complex lake-river interactions: Poyang Lake (China). Water, 2015, 7(5): 1991-2012.

[20] Li YL, Yao J. Estimation of transport trajectory and residence time in large river-lake systems: Application to Poyang Lake (China) using a combined model approach. Water, 2015, 7(10): 5203-5223.

[21] Zhang Q, Werner AD. Hysteretic relationships in inundation dynamics for a large lake-floodplain system. Journal of Hydrology, 2015, 527: 160-171.

[22] Monsen NE, Cloern JE, Lucas LV et al. A comment on the use of flushing time, residence time, and age as transport time scales. Limnology and Oceanography, 2002, 47: 1545-1553.

[23] Takeoka H. Fundamental concepts of exchange and transport time scales in a coastal sea. Continental Shelf Research, $1984, \mathbf{3}(3)$ : 311-326.

[24] Dyer KR. Estuaries: A physical introduction. London: John Wiley and Sons Ltd, 1997.

[25] Cucco A, Umgiesser G. Modeling the Venice Lagoon residence time. Ecological Modelling, 2006, 193(1/2) : $34-51$.

[26] Nyberg L, Rodhe A, Bishop K. Water transit times and flow paths from two line injections of $3 \mathrm{H}$ and $36 \mathrm{Cl}$ in a microcatchment at Gardsjon, Sweden. Hydrological Processes, 1999, 13: 1557-1575.

[27] Botter G, Bertuzzo E, Rinaldo A. Catchment residence and travel time distributions: The master equation. Geophysical Research Letters, 2011, 38, L11403. DOI: 10.1029/2011GL047666.

[28] Razmi AM, Barry DA, Lemmin U et al. Direct effects of dominant winds on residence and travel times in the wide and open lacustrine embayment: Vidy Bay (Lake Geneva, Switzerland). Aquatic Sciences, 2014, 76(1) : 59-71.

[29] Dabrowski T, Hartnett M. Modelling travel and residence times in the eastern Irish Sea. Marine Pollution Bulletin, 2008, 57: $41-46$.

[30] DHI. MIKE 21 flow model: Hydrodynamic module user guide. Hørsholm: Danish Hydraulic Institute Water and Environment, 2014.

[31] DHI. MIKE 21 flow model FM: Transport module user guide. Hørsholm: Danish Hydraulic Institute Water and Environment, 2014.

[32] Dabrowski T, Hartnett M, Olbert AI. Determination of flushing characteristics of the Irish Sea: A spatial approach. Computers \& Geosciences, 2012, 45: 250-260.

[33] Wan Y, Qiu C, Doering P et al. Modeling residence time with a three-dimensional hydrodynamic model: Linkage with chlorophyll a in a subtropical estuary. Ecological Modelling, 2013, 268: 93-102.

[34] Wu Z, He H, Cai Y et al. Spatial distribution of chlorophyll a and its relationship with the environment during summer in Lake Poyang: A Yangtze-connected lake. Hydrobiologia, 2014, 732: 61-70.

[35] Gao Guiqing, Ruan Renzeng, Ouyang Qiulin. Water quality status and change trend in Poyang Lake. Jounal of Nanchang Institute of Technology, 2010, 29(4) : 50-53. [高桂青, 阮仁增, 欧阳球林. 鄱阳湖水质状况及变化趋势分析. 南昌 工程学院学报, 2010, 29(4): 50-53.]

[36] Liu X, Li YL, Liu B et al. Cyanobacteria in the complex river-connected Poyang Lake: Horizontal distribution and transport. Hydrobiologia, 2016, 768(1) : 95-110. 